\title{
Role of CB-NAAT in diagnosing Mycobacterial tuberculosis and rifampicin resistance in tubercular peripheral lymphadenopathy
}

\author{
Singh KG ${ }^{1}$, Tandon $S^{2}$, Nagdeote $S . T^{3}$, Sharma $K^{4}$, Kumar $A^{5}$ \\ ${ }^{1}$ Dr. Krishna Gopal Singh, P.G Resident, ${ }^{2}$ Dr Sanjay Tandon, Professor and Head, ${ }^{3}$ Dr S.T Nagdeote, Professor, \\ ${ }^{4}$ Dr. Kapil Sharma, Assistant Professor, ${ }^{5}$ Dr. Ajay Kumar, P.G Resident, all authors are affiliated with Department of \\ Pulmonary Medicine, People's College of Medical Sciences \& RC, Bhanpur, Bhopal, MP, India.
}

Address for Correspondence: Dr Sanjay Tandon, Professor and Head, Department of Pulmonary Medicine, Peoples College of Medical Sciences \& RC, Bhanpur, Bhopal. Email: pulmedph@gmail.com

\begin{abstract}
Introduction: Diagnosis of Extra Pulmonary TB (EPTB) remains a challenge. This study assessed the applicability of CB-NAAT in early diagnosis of EPTB, especially peripheral lymphadenopathy and early identification of MDR-TB in TB lymphadenopathy. Material \& Method: This was a prospective, cross sectional study, carried out on all patients of age group 10 to 80 years, who were suspected cases of tubercular peripheral lymphadenopathy, visiting the OPD of the Department of Pulmonary Medicine, People's Hospital, Bhopal, over a period of 1 year 6 months. FNA of peripheral lymph nodes of 57 patients was sent for cytology and CB-NAAT. Result: This study showed that statistical difference in diagnostic yield of FNAC (47/57 i.e. 82.4\%) and CB-NAAT (44/57 i.e. 77.19\%) in tubercular peripheral lymphnode sample was not significant. This implies that there was not much difference in these two test results. There was no rifampicin resistance in the population studied. Conclusion: CB-NAAT did not diagnose more cases of TB-LN than FNAC.
\end{abstract}

Keywords: Extra Pulmonary Tuberculosis (EPTB), Cartridge Based Nucleic Acid Amplification Test (CB-NAAT), Multi Drug Resistant Tuberculosis (MDR-TB), Fine needle aspiration cytology (FNAC).

\section{Introduction}

Tuberculosis (TB) is an important health problem in low and middle income countries and remains a key challenge to public health. Our ability to tackle this disease has been severely hampered by inadequate diagnostic assays [1]. No single diagnostic test is satisfying to all the demand of rapid, affordable and easy confirmatory test. A lot of research has taken place in the direction for diagnosis and treatment of pulmonary TB.

However, diagnosis of Extra Pulmonary TB (EPTB) still remains challenging, since the number of Mycobacterium Tuberculosis Bacilli (MTB) present at the site of diseased tissue is often low and clinical specimens of deep-seated organs are difficult to obtain [2].

Manuscript received: $8^{\text {th }}$ March 2017

Reviewed: $17^{\text {th }}$ March 2017

Author Corrected: 24 $4^{\text {th }}$ March 2017

Accepted for Publication: $31^{\text {st }}$ March 2017
Histology is time-consuming and remains difficult to undertake and establish the diagnosis of TB with high specificity [2]. Fine Needle Aspiration Cytology (FNAC) to diagnose TB obviates the need for lymph node excisional biopsy. Since, FNAC carries a high degree of accuracy; it is used in diagnosis at the initial step as well as in the follow-up post treatment of tubercular peripheral lymphadenopathy[3].

When compared, Excisional biopsy has the highest sensitivity, whereas FNA is less invasive, pain free, OPD procedure with no morbidity. It has been available for nearly two decades and serves as the first-line diagnostic technique in superficial TB lymphadenopathy with sensitivity and specificity of $79 \%$ and 94\% respectively [4]. One cannot completely rely on FNAC, which is the mainstay of diagnosis, because of contamination, subjectivity of the test, failure to differentiate between different causes of granuloma, no 
role in drug resistant $\mathrm{TB}$ and inefficient diagnosis amongst the immune-compromised patients. Thus the need for new diagnostic tool arose.

Molecular diagnostic methods seem to hold the key to the future of better and efficient diagnosis of EPTB. In order to obtain faster results, Nucleic Acid Amplification Test (NAAT) is being increasingly used worldwide for the rapid diagnosis of TB. Genotypic methods have considerable advantages, in terms of scaling up the programmatic management and surveillance of Multi Drug Resistant-TB (MDR-TB), offering quicker diagnosis, standardized testing, the potential for high output and having fewer requirements for ensuring laboratory bio safety [5]. Several Nucleic Acid Amplification Technology (NAAT), tests have been developed that rapidly detect $M$. tuberculosis DNA in patient samples and look for DNA changes that make M. tuberculosis drug-resistant.

Cartridge Based Nucleic Acid Amplification test (CBNAAT), is an automated DNA test that detects M. tuberculosis and rifampicin resistance (an indicator of MDR-TB) within two hours for the investigation of patients who might have TB [5]

However, due to paucity of data, more studies are needed to recommend CB-NAAT testing as the initial test in suspected TB lymphadenopathy. In this study, we evaluated the performance of CB-NAAT for the diagnosis of TBLN on fine needle aspirates [6]. This study aimed to assess the applicability of CB-NAAT in early diagnosis of tubercular peripheral lymphadenopathy and early identification of MDR-TB (rifampicin resistance) in lymph node TB.

\section{Material \& Methods}

Study design- This was a prospective, cross sectional study.

Inclusion criteria- This study was carried out in all patients of age group 10 to 80 years, who were suspected cases of Tubercular peripheral lymphadenopathy and diagnosed TB by cytology and excisional biopsy, and who visited Pulmonary Medicine OPD of People's Hospital, Bhopal, between November, 2014 and April, 2016.

Exclusion Criteria- Cases that were not included in the study were:
Cases already diagnosed with MDR/XDR-TB by CBNAAT, concurrent/concomitant reason for admission, active or suspected malignancy, terminal disease with poor prognosis, non-cooperative patients, patients not willing to give consent for study and patients with past history of TBLN treatment.

Data source: Who visited Pulmonary Medicine OPD of People's Hospital, Bhopal, between November, 2014 and April, 2016. Peoples Hospital is a teaching institute and a reference centre for the urban and rural population in Central India.

Study size- A total of 57 patients were taken for this study.

Statistical method- The data obtained was compiled systematically and statistically analyzed. Statistical procedures were carried out in two steps: Data compilation and presentation and Statistical analysis.

Statistical analysis was done using Statistical Package of Social Science (SPSS Version 15; Chicago Inc., USA) and data comparison was done by applying specific statistical tests to find out the statistical significance of the comparisons. Quantitative variables were compared using mean values and qualitative variables using proportions and inconsistencies were checked against the raw data.

The McNemar test was also used to compare the smear results among the FNAC and CB-NAAT for assessing statistical significance.

Method- A total of 57 patients were taken for this study. Data for the study was collected using convenient sampling techniques after obtaining written consent. The data was collected with face to face interview of the patients, using a pre-designed questionnaire, which included patient's identification data and socioeconomic status.

The skin of the patient, over the suspected lymph node to be aspirated was cleaned and disinfected using spirit swabs. The lymph node was then fixed between the index finger and the thumb of the left hand. Following this, An 18 gauge needle attached to $10 \mathrm{ml}$ syringe was introduced into the lymph node. Vacuum was created in the syringe by pulling back the plunger and the needle was carefully moved in different directions to dislodge the material. When adequate material was aspirated into 
the syringe, the suction was gently released to equalize the pressure which prevented the sucking of aspirated material into the barrel of the syringe.

One part of this aspirated material was put in a sterile container and sent for CB-NAAT and the other part was smeared on 2-3 slides. One smear slide was fixed in 95\% ethyl alcohol for staining with HaematoxyleneEosin and Papanicolaou stain and the other was air dried for Giemsa staining. Few slides were kept aside for special stains wherever required e.g. AFB for TB etc. The slides were examined under the microscope for visualization of granulomas and AFB. All patients who had a negative TB cytology on FNA sample underwent excisional biopsy of TB lymphnode.

Ethical approval- Study was approved by the Institutional Ethics and Research Committee, People's Medical College, Bhopal. An informed written consent in the presence of witnesses was taken from all the volunteer patients who participated in the study, before conducting the interview and sample collection. Written informed consent was taken all participants.

\section{Results}

All suspected patients with tubercular peripheral lymphadenopathy between the age group 10 to 80 years who visited the OPD of Pulmonary Medicine of People's Hospital were included in the study.

Table-1: Demographic and characteristics of study population.

\begin{tabular}{|c|c|}
\hline Total no of patients (n) & 57 \\
\hline Age range (years) & $10-80$ \\
\hline Male/Female ratio & $3: 2$ \\
\hline Urban/Rural distribution & $7: 3$ \\
\hline No. of patients who underwent FNAC & 57 \\
\hline No. of patients who underwent CB-NAAT & 57 \\
\hline No. of patients who underwent lymph node biopsy & 10 \\
\hline
\end{tabular}

Total number of patients in our study was 57 in which FNA for cytology and CB-NAAT was done. 10 patients which were cytology negative underwent excisional biopsy. Majority of the patients were from urban areas and of age group 20-30 years and males were preponderant. No patient refused the test.

Table-2: Comparison between investigations.

\begin{tabular}{|c|c|c|c|}
\hline & FNAC positive & FNAC negative & Total \\
\hline CB-NAAT positive & 43 & 1 & 44 \\
\hline CB-NAAT negative & 4 & 9 & 0 \\
\hline Rifampicin resistant & 0 & 13 & 10 \\
\hline Biopsy positive TBLN & Not done & 10 & 57 \\
\hline $\begin{array}{c}\text { Response to ATT } \\
\text { treatment }\end{array}$ & 47 & & 0 \\
\hline
\end{tabular}

CB-NAAT was positive from lymph node in 43 out of the 47 FNAC positive patients. In 10 patients in whom FNAC was negative, CB-NAAT did not diagnose TBLN in 9 of them. All of 10 FNAC patients negative by cytology for TBLN, showed caseating granulomas consistent with TB on histopathological examination of LN following Excisional Biopsy.

Rifampicin resistance was not seen in any patient. All of the 57 patients responded to ATT and were declared cured in 6 to 9 months of standard short course regime.

The p-value of CB-NAAT for diagnosing tubercular lymphadenopathy is significant a.e $\mathrm{p}<.05$.

The McNemar test was applied to the results of FNAC and CB-NAAT. However the statistical difference was not significant. This implies that there was not much difference in these two test results. 


\section{Discussion}

A lot of research has taken place in the direction of tests for diagnosis and treatment of pulmonary TB. However, there is still a large lacuna in research for diagnosis of extra pulmonary TB. This study of 57 cases was conducted to look into the role of CB NAAT in diagnosing tubercular lymphadenopathy and rifampicin resistance in tubercular peripheral lymphadenopathy.

Out of a total of 57 cases, FNAC could diagnose tubercular cytology in 47 cases $(82.4 \%)$. In remaining 10 patients in whom FNAC was negative for TB cytology, histopathology following excisional biopsy of lymph node was positive for TB lymphadenopathy.

CB-NAAT done in these 10 patients before excisional biopsy on FNA sample was positive in only one patient indicating that CB-NAAT is mostly negative for MTB if cytology is also negative for TBLN. All the 10 slides which were negative by cytology were reviewed and were found to have good material on slides. Hence, inadequate sampling of the lymph nodes could not have been the reason for negative reports.

Similar observations were found in other studies. Malakar et al did a study in a series of 60 patients in which FNAC was positive in 54 patients (90\%) [4]. In a study conducted by Narang et al it was found that cytology confirmed the histopathological diagnosis in 51 of 60 cases $(85 \%)$ [11]. In 9 cases cytological diagnosis was not in accordance with histopathological diagnosis.

In our study CB-NAAT was positive for MTB in 44 $(77 \%)$ out of 57 patients with cytologically and histopathologically proven TB lymphadenopathy. MTB was not detected by CB-NAAT in $23 \%$ cases of tubercular peripheral lymphadenopathy.

CB-NAAT was found to be negative for MTB in almost all cases in which cytology was negative but histopathology was positive.

In a study by Ligthelm et al in 2011 South Africa, CBNAAT was positive in $96 \%$ (29 out of 30 patients) of the cases with tubercular peripheral lymphadenopathy in FNA sample [6]. In a study conducted by Claudia M denkinger et. al found that sensitivity of CB-NAAT is $83.1 \%$ and specificity of CB-NAAT is $93.6 \%$ as compare to TBLN culture [16].
Meta analysis done by Ji Yeon et. al in 2015 Seoul, Korea found that, sensitivity of CB-NAAT is $80 \%$ for diagnosing tubercular peripheral lymphadenopathy [2].

Our study, therefore, showed that CB-NAAT could not diagnose TB lymphadenopathy in sample negative by cytology. Hence CB-NAAT should not be used to diagnose TB lymphadenopathy in patients with negative cytology in FNAC sample.

In my study CB-NAAT did not detect rifampicin resistance in any of the patients diagnosed with $\mathrm{TB}$ lymphadenopathy. In a study by Ligthelm et al small number of Rifampicin-resistant cases identified i.e 1 of $2(50 \%)[2]$.

Mulualem Tadesse et al in 2015 found that, CB-NAAT detect Rifampicin resistant was low in TBLN, 4.7\% (4/86) in Central African patients [6]. Also it was observed that Rifampicin resistance in TB peripheral lymphadenopathy is very low and warrant high degree of suspicion and strong laboratory back up [23]. In addition CB-NAAT is more expensive as compared to cytology.

McNemar test was applied to the results of FNAC and CB-NAAT. However the statistical difference was insignificant. This implies that there was not much difference between these two test results.

\section{Conclusion}

To conclude, this study showed that as compared to FNAC, CB-NAAT is not a very significant test for diagnosis of Tubercular peripheral lymphadenopathy, especially in FNAC negative cytology. Moreover, it is not very cost effective, demands a trained man force, 24-hour electricity backup, regular maintenance and calibration. More research and refinement is needed for CB-NAAT, as a future diagnostic tool.

Hence we recommend that CB-NAAT should not be used to diagnose TB lymphadenopathy, where FNA cytology is negative However, it may be used to diagnose Rifampicin resistance in suspected cases. A more robust and simpler technology which is welladapted to low-resource settings is still needed.

Funding: Nil, Conflict of interest: None Permission of IRB: Yes 


\section{References}

1. Ardizzoni E, Fajardo E, Saranchuk P, Casenghi M, Page AL, Varaine F, Kosack CS, Hepple P. Implementing the Xpert@ MTB/RIF Diagnostic Test for Tuberculosis and Rifampicin Resistance: Outcomes and Lessons Learned in 18 Countries. PLoS One. 2015 Dec 15;10(12):e0144656.doi:10.1371/journal.pone.0144656. eCollection 2015.

2. Lee JY. Diagnosis and treatment of extrapulmonary tuberculosis.Tuberc Respir Dis(Seoul).2015Apr;7 8 (2): 47-55.doi:10.4046/trd.2015.78.2.47.Epub 2015Apr 2.

3. Aljafari AS, Khalil EA, Elsiddig KE, El Hag IA, Ibrahim ME, Elsafi ME, Hussein AM, Elkhidir IM, Sulaiman GS, Elhassan AM. Diagnosis of tuberculous lymphadenitis by FNAC, microbiological methods and PCR: a comparative study. Cytopathology. 2004 Feb;15 (1):44-8.

4. Louis J.Ligthelm Xpert MTB/RIF for Rapid Diagnosis of Tuberculous Lymphadenitis from FineNeedle-Aspiration Biopsy Specimens. Journal of clinical microbiology, Nov. 2011, p. 3967-3970 Vol. 49, No. 110095-1137. doi:10.1128/JCM.01310-11.

5. Catherina CB, Rapid molecular detection of TB and Rifampicin resistance, NEJM, 2010, Sep; 363:11. Doi: 10. 1056/NEJMoa0907847.

6. Tadesse M, Abebe G, Abdissa K, Aragaw D, Abdella K, Bekele A, Bezabih M, Apers L, de Jong BC, Rigouts L. GeneXpert MTB/RIF Assay for the Diagnosis of Tuberculous Lymphadenitis on Concentrated Fine Needle Aspirates in High Tuberculosis Burden Settings. PLoS One. 2015 Sep 14;10(9):e0137471. doi: 10.1371/ journal. pone.0137471. eCollection 2015.

7. Dandapat MC. Diagnosis of tubercular lymphadenitis by fine needle aspiration cytology. Indian $\mathrm{J}$ Tuberc 1987; 37: 139-142.D

8. Golden MP, Vikram HR. Extrapulmonary tuberculosis: an overview. Am Fam Physician. 2005 Nov 1;72(9):1761-8.
9. D Vijay sekaran. Extra pulmonary tuberculosis an review. Indian journal of Pediatrics, 2006; vol.23.

10. Nayak S, Acharjya B. Mantoux test and its interpretation. Indian Dermatol Online J. 2012 Jan;3(1): 2-6. doi: 10.4103/2229-5178.93479.

11. Zuber Ahmed. Antimicrobial drug resistance. Journal, Indian Academy of Clinical Medicine 2003; Oct. Vol. 4, No. 4.

12. R. K. Narang. Place of fine needle aspiration cytology in the diagnosis of lymphadenopathy. Ind. J. Tub. 1990, 37, 29.

13. Malakar ILN. Aspiration Cytology for the Diagnosis of Tuberculous Lymphadenopathies:A Five-year Study. Ind. J. Tub., 1991 38, 17.

14. Denkinger CM, Schumacher SG, Boehme CC, Dendukuri N, Pai M, Steingart KR. Xpert MTB/RIF assay for the diagnosis of extrapulmonary tuberculosis: a systematic review and meta-analysis. Eur Respir J. 2014Aug;44(2):435-46.doi:10.1183/09031936.0000 7814. Epub 2014 Apr 2.

15. Mark pinto Nicole Molecular method in node. Current Respiratory Medicine Reviews,2013,Vol.9,No3

16. Pamra S. Cervical lymphadenopathies. Indian J Tuberc 1987; 96-100.

17. Ergete W. Acid fast bacilli in aspiration smears from tuberculous patients. Ethiop J Health Dev 2000; 14 (1): 99-104.

18. Purohit MR. Gender differences in the clinical diagnosis of tuberculous lymphadenitis - a hospitalbased study from central India. International Journal of Infectious Diseases 2009 Sep;13(5): 600-05.s

19. Rajsekaran S. Tuberculous cervical lymphadenitis in HIV positive and negative patients. Indian J Tuberc 2001; 48: 201-4.

20. Ahmad SS. Incidence of tuberculosis from study of fine needle aspiration cytology in lymphadenopathy and acid fast staining.Ind J CommunityMedicine2005;30 (2)

\section{How to cite this article?}

Singh KG, Tandon S, Nagdeote S.T, Sharma K, Kumar A. Role of CB-NAAT in diagnosing Mycobacterial tuberculosis and rifampicin resistance in tubercular peripheral lymphadenopathy. Int J Med Res Rev 2017;5(03):242-246 doi:10.17511/ijmrr. 2017.i03.05. 